\title{
Sex Differences in the Brain: The Not So Inconvenient Truth
}

\author{
Margaret M. McCarthy, ${ }^{1}$ Arthur P. Arnold, ${ }^{2}$ Gregory F. Ball, ${ }^{3}$ Jeffrey D. Blaustein, ${ }^{4}$ and Geert. J. De Vries ${ }^{4}$ \\ ${ }^{1}$ Department of Pharmacology, University of Maryland School of Medicine, Baltimore, Maryland 21201, ${ }^{2}$ Department of Integrative Biology and Physiology, \\ University of California Los Angeles, Los Angeles, California 90095, ${ }^{3}$ Department of Psychological and Brain Sciences, Johns Hopkins University, Baltimore, \\ Maryland 21218, and ${ }^{4}$ Center for Neuroendocrine Studies, University of Massachusetts, Amherst, Massachusetts 01003
}

\section{Introduction}

In 2001 the Institute of Medicine, a branch of the National Academy of Sciences in the U.S.A., concluded that many aspects of both normal and pathological brain functioning exhibit important yet poorly understood sex differences (Wizemann and Pardu, 2001). Ten years later, the National Institute of Mental Health convened a workshop titled Sex Differences in Brain, Behavior, Mental Health and Mental Disorders and concluded (1) there is a paucity of research examining sex differences at a neurobiological and mechanistic level; (2) there are pervasive sex differences in the brain, and (3) there is a need for more neuroscientists to incorporate sex as a variable in experimental designs (National Institute of Mental Health, 2011).

Several other government funding agencies in the United States and Europe have either explicitly or implicitly endorsed the study of the impact of sex and/or gender as important research goals to increase our understanding of normal brain development, adolescence, reproduction and

Received 0ct. 25, 2011; revised Jan. 9, 2012; accepted Jan. 11, 2012.

The authors of this article are: a member of the Society for Neuroscience Program Committee (M.M.M.), founding president of the Society for Behavioral Neuroendocrinology (SBN) and Editor-in-Chief of Biology of Sex Differences (A.P.A.), past-president of the SBN (G.F.B.), current president of SBN and Editor-in-Chief of Endocrinology (J.D.B.), and past-president of SBN and president-elect of the Organization for the Study of Sex Differences (G.J.D.).

Correspondence should be addressed to Dr. Margaret M. McCarthy, University of Maryland School of Medicine, 655 W. Baltimore Street, Baltimore, MD 21201. E-mail: mmccarth@umaryland.edu.

DOI:10.1523/JNEUROSCI.5372-11.2012

Copyright $\odot 2012$ the authors $\quad 0270-6474 / 12 / 322241-07 \$ 15.00 / 0$ aging, as well as pathologies in behavior involving socialization, ingestion, sleep, and substance abuse. There are important sex differences in cognitive and emotional responses relevant to learning and memory, language, fear, anxiety and nociception, as well as the risk and consequences of traumatic brain injury, stroke, and the neurodegenerative diseases Parkinson's, Alzheimer's, ALS, and Huntington's. Neurological disorders such as dyslexia and stuttering are three to four times more frequent in boys than girls, and attention deficit hyperactivity disorder is diagnosed 10 times more often in boys. In the arena of mental health, gender is also a potent predictor of the relative risk of developing autism or autism spectrum disorder, both of which are up to four times more prevalent in males, as is early-onset schizophrenia, with more severe symptoms reported in boys. Conversely, major depressive disorder, anxiety, and panic disorders are almost two times as frequently diagnosed in women compared with men, while anorexia bulimia is three times as prevalent and anorexia nervosa is a disturbing 13 times more frequent in women (De Vries and Simerly, 2002; Simerly, 2002; Morris et al., 2004; Baron-Cohen et al., 2005; Cahill, 2006; Forger, 2009; McCarthy et al., 2009; Abel et al., 2010; Jazin and Cahill, 2010; McCarthy and Arnold, 2011). A potentially informative difference in the pattern of gender bias in relative risk of mental illness is the preponderance of developmental onset disorders in males versus the higher frequency of adult onset disorders in females. Despite these profound differences, males remain the research subject of choice in neuroscience (Beery and Zucker, 2011).

When we attempt to encourage our neuroscience friends and colleagues to consider the value of studying sex differences, we often hear "after I understand the phenomenon in males, I'll check whether it's there in females," or "I would be interested in looking for sex differences but can't afford to double my $N$," and, last, "I would have to control for the phases of the estrous/menstrual cycle in females and that just seems too difficult and a waste of resources." In our view, what most deters investigators from including females in their studies are misconceptions: misconceptions that it is difficult to do it right, and misconceptions of the value of comparing males and females, with many neuroscientists thinking they are not likely to learn anything useful, much less make novel discoveries. The purpose of this Toolbox article is to dispel those myths and provide simple guidelines for scientifically sound investigation of sex differences in the brain.

\section{Classification of sex differences}

There is no clear biological divide regarding types of sex differences, but defining basic nonexclusive categories provides guidance for experimental design. Thus we propose subdividing sex differences into three types (Table 1 ). The first reflects absolute sexual dimorphism, meaning that a particular endpoint (behavioral, physiological, or morphological) has two forms, one found either exclusively or predominantly in males and the other in females. The divergence is sufficiently 
Table 1. Operationally defined "types" of sex differences

\begin{tabular}{|c|c|c|}
\hline Category & Description & Examples \\
\hline Type I—sexual dimorphism & $\begin{array}{l}\text { Endpoint consists of two forms, one more prevalent in males and the other } \\
\text { more prevalent in females. Endpoint may be present in one sex and } \\
\text { absent in the other. }\end{array}$ & $\begin{array}{l}\text { Copulatory behavior, bird song, nurturing, postpartum aggression, } \\
\text { courtship displays }\end{array}$ \\
\hline Type II-sex differences & $\begin{array}{l}\text { Endpoint exists on a continuum and average is different between males } \\
\text { and females. }\end{array}$ & $\begin{array}{l}\text { Pain thresholds, food preferences and intake, odor detection, fear, anxiety, } \\
\text { learning, memory, stress responding, sensory processing }\end{array}$ \\
\hline $\begin{array}{l}\text { Type III- sex convergence and } \\
\text { divergence }\end{array}$ & $\begin{array}{l}\text { Endpoint is the same in males and females but neural underpinnings are } \\
\text { different. Alternatively, a sex difference may appear only in response to } \\
\text { a challenge such as injury or stress. }\end{array}$ & Parental behavior, problem solving strategies, response to stress \\
\hline
\end{tabular}

Prior to embarking on the study of a particular sex difference it is useful to consider the nature of the difference. The distinctions presented in this table provide basic guidelines but are not exclusive and in many cases are species specific. Some sex differences may not fit neatly into any of these categories and certainly there are many that have yet to be discovered.

great that it is categorically distinct from other sex differences. Included in this type are traits that are present in one sex but missing in the other. Absolute sexual dimorphisms in behavior are often directly associated with reproduction and include, for example, male bird courtship singing, male-specific courtship displays, territory defense, copulatory behavior, nurturing and postpartum aggression in some species. Likewise, there are sex differences in the number, shape, and biochemical make-up of participating neurons and glial cells that either correlate with sex differences in behavior or, in rarer instances, have been more directly linked to such.

The second type of sex difference is one that exists along a continuum in which males or females can fall at any point but the average differs between the sexes. Stress and anxiety responses, food preferences and intake, learning and memory, social behavior, somatosensory thresholds, pain sensitivity, olfaction and verbal recall are all examples of traits that differ on average between males and females but also show varying degrees of overlap. Sex differences in this category may or may not be directly related to reproduction. For example, genome-wide association studies examining risk for major depressive disorder or bipolar disorder implicated a gene that codes for the dominant isoform of the L-type voltage-gated calcium channel in the brain $\left(\mathrm{Ca}_{\mathrm{v}} 1.2\right)$. In a case of reverse translation, mice engineered to be haploinsufficient for $\mathrm{Ca}_{\mathrm{v}} 1.2$ exhibited increased anxiety and depresssive-like behaviors on a battery of tests, but the severity of the effect was greater in females than in males. This insight led to further study in humans and identified two intronic single-nucleotide polymorphisms that are associated with increased prevalence of mood disorders in females only (Dao et al., 2010), demonstrating the power of both animal studies and exploration of sex differences.

The third type is perhaps the most unusual in that it involves instances where the sexes either converge to the same endpoint or start the same and diverge in response to some challenge. For instance, there may be no obvious sex difference in a particular behavior, but the underlying neurophysiology regulating the behavior can be markedly different in males and females. Thus, factors in one sex may offset the effects of the other so that the result is to make the sexes more similar. For example, in some mammalian species fathers show robust parental care, indistinguishable from females in many ways. The neural underpinnings of this behavior, however, are different in males versus females because males do not experience the hormonal milieu of pregnancy, parturition, and lactation that is critical to the induction of maternal care by mammalian females. In one case, the biparental prairie vole, the neurocircuitry for vasopressin appears to have been selectively altered in males to promote care-giving behavior (De Vries, 2004). Another example is the different strategies used by males and females to solve the same spatial learning problem. Males and females can learn the task equally well, but the external constraints imposed on the task affects the strategy used and can create a sex difference in performance, but not ability. This has been well characterized for the Morris Water Maze spatial learning test where both pre-test conditioning and the route used to gain access to the platform (females hug the walls more) impacts the outcome measures of the test in sex-specific ways (Perrot-Sinal et al., 1996; Beiko et al., 2004). In contrast to these examples of convergence, there are also so-called latent sex differences, meaning those that appear only under certain conditions such as following injury, or exposure to environmental toxins or to physical and psychological stressors. In other words, the sexes appear to be similar at baseline, but in fact have different neurochemical underpinnings that result in different vulnerabilities or divergent outcomes in response to the same stressor. A clear demonstration of this is found in the work on eye-blink conditioning in rats. Not only do male rats improve their performance in response to stress, whereas the female's learning is impaired, but stress induces opposite effects in the two sexes on dendritic spine formation of CA1 pyramidal neuron dendrites (Shors et al., 2001). In this instance, if only males were studied, the conclusion would be that stress is good for associative learning, whereas if only females were studied, the conclusion would be the opposite.

\section{Causes of sex differences}

The study of sex differences in the brain can trace its origins back to the mid1800s, when Arnold Berthold removed the testes from roosters and noted that they became less aggressive and lost interest in hens. He concluded that "The testis acts on the blood, and the blood acts on the whole organism". The modern era of behavioral endocrinology began with the pioneering work of Frank A. Beach in the 1940 s but is more clearly demarcated by the iconic report of Phoenix, Goy, Gerall and Young in 1959, which articulated the Organizational/Activational hypothesis of hormone action (Becker et al., 2002). This theory states that gonadally derived steroid hormones early in development organize the substrate controlling adult sexual behavior, creating permanent sex differences in neural circuits, and that this organized substrate is then activated by the sex-specific hormonal milieu of adulthood. The same principles were applied to sexual differentiation of bird song some 15 years later and included the discovery of highly dimorphic song control nuclei (Arnold et al., 1996; Wade and Arnold, 2004). These observations spawned a cottage industry of research into the hormonal and neural control of reproductive physiology and behavior that has revealed numerous sex differences at every level of organization in the brain (Pfaff et al., 2002). Yet the field has remained a subdiscipline within neuroscience-interesting, but not mainstream. 


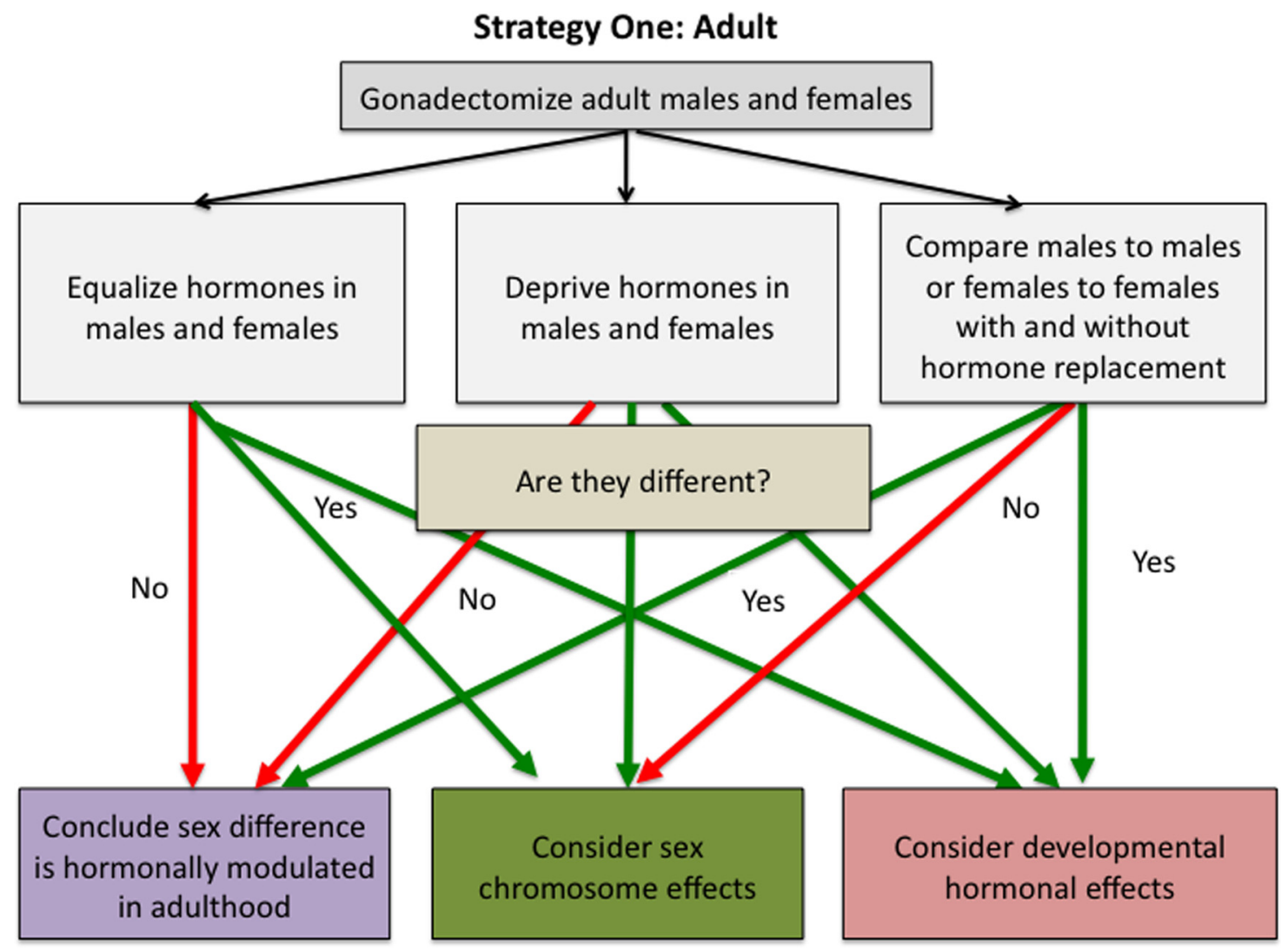

Figure 1. Strategy for discerning the basis of a sex difference starting with adult difference in the activational actions of testicular versus ovarian hormones. Because sex differences caused by gonadal hormones are the majority and are easier to study than those caused by sex chromosome effects, a simple way to start is to remove the gonads of males and females as adults and then perform various comparisons. If the sex difference persists when all gonadal hormones are removed, the source of the sex difference may be organizational effects of steroids during development or differences in sex chromosome complement. A second useful approach is to provide exogenous hormones to gonadectomized animals. If evidence suggests a sex difference is male biased, then starting by mimicking the hormonal status of males in both sexes (i.e., both receive testosterone) is probably the best approach. Conversely, if there is reason to believe a sex difference is female biased, then begin by providing both sexes a female typical hormonal profile (i.e., both receive estradiol or estradiol plus progesterone). If equating hormone levels abolishes the sex difference, the sex difference is due to adult differences in gonadal steroid levels. If, however, the sex difference persists, one should again consider the effects of developmental hormone exposure or sex chromosome complement.

\section{Sex differences come in different shapes and forms with divergent origins}

The most pervasive sex difference in the brain is the self-evident, but rarely acknowledged, fact that every neuron, glia, or other cell type carries either the complement of male chromosomes (XY) or female chromosomes (XX), but not both (Arnold and Burgoyne, 2004). Long ignored as an important factor, the importance of genotype versus gonadal phenotype is beginning to emerge. This is in large part due to the development of genetically modified mice in which gonadal phenotype and chromosomal genotype can be separated (De Vries et al., 2002). Thus, any modern study of sex differences in the brain should first consider whether its cause is hormonal, genetic, or both. It is likely that the majority of sex differences in the brain are caused by gonadal hormones, acting in the adult (different effects of ovarian and testicular hormones), or early in development (especially effects of testicular hormones). Experiments ma- nipulating hormones are the easiest in animals, and are usually used first (see below). If hormones do not explain the sex differences, then one can consider further experiments to evaluate the different effects of XX versus XY sex chromosome complement.

All of the factors mentioned above cause differences in morphology and physiology that in turn may lead to different interactions with the environment. Therefore, the imposition of an external variable can induce additional sex differences by impacting only one sex, thereby making it different from the other sex, or by driving the two sexes in the opposite direction along a continuum.

\section{Where to begin?}

A central part of explaining sex differences is to identify the factors that makes a trait different in males and females. A good first experiment is to ask if the sex difference is caused by gonadal hormones, as hormones induce the large majority of sex differences. You can either ask, is my adult sex difference determined by steroid hormones in adulthood (Fig. 1)? Or, is my adult sex difference the consequence of developmental exposure to steroids (Fig. $2)$ ? The emphasis on development stems from the overwhelming evidence supporting an early sensitive period, usually perinatal, for the organizational or enduring effects of hormones. Puberty should be considered as well, as it has recently been recognized as an additional sensitive period for enduring effects of hormones (Sisk and Zehr, 2005). Regardless of the timing of the sensitive period, the approach you take depends on a number of considerations, including the species you are studying and the question you are asking. Moreover, in humans one is constrained by the inability to manipulate hormones except in adulthood, or to assess intracerebral steroid concentrations. Thus, one has to rely instead on serum or saliva assays, indirect markers of developmental steroid exposure (Breedlove, 
Strategy Two: Neonate

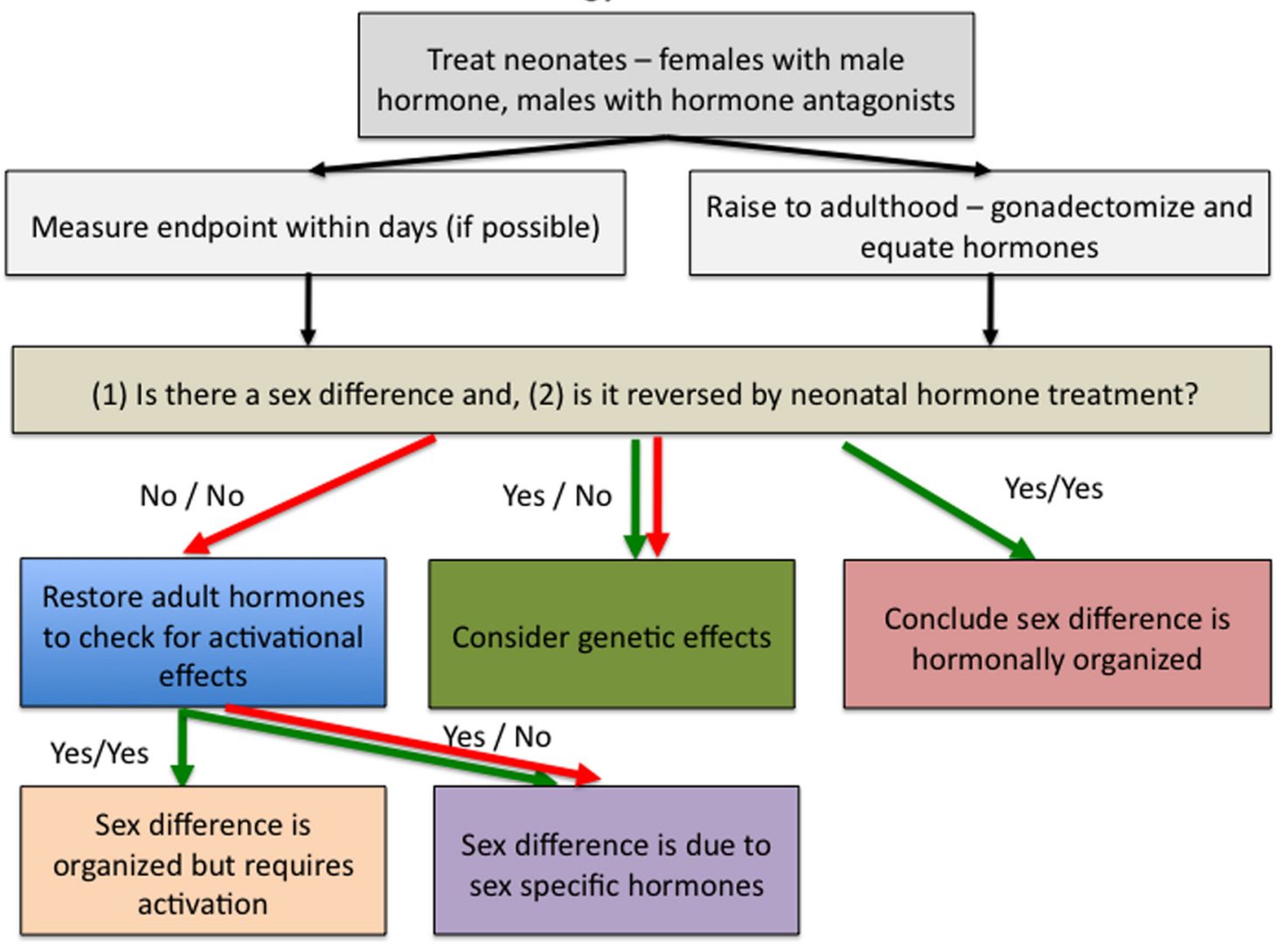

Figure 2. Strategy for discerning the basis of a sex difference starting with the neonate. Because sex differences in the action of gonadal hormones cause permanent sex differences in brain circuits, for some endpoints it is acceptable to test immediately for such fetal or neonatal effects. Following well established protocols available in the work of Becker et al. (2005), neonatal rats and mice can be readily treated with steroid hormones. Comparing males, females, males treated with blockers of steroid hormone action, and females administered masculinizing doses of steroid hormones tests whether a sex difference in hormone action during development causes the sex difference. If a particular endpoint cannot be measured in the neonate, animals are raised to adulthood. Because neonatal treatment can alter adult levels of gonadal hormones by altering the hypothalamic-pituitary-gonadal axis, any effect of neonatal treatment could be interpreted as a change in brain circuits or an altered hormonal profile if the gonads remain. A common method to distinguish between these possibilities is to remove group differences in hormone levels in adulthood by gonadectomy and, if possible, to compare males and females on the relevant behavioral or neural variable. If the sex difference persists, this suggests either a purely organizational effect with no role for hormonal activation, or a genetic basis for the sex difference. In many cases, however, the endpoint under study will not be evident in conditions in which hormones are completely lacking. In these instances the appropriate approach is treating males and females with similar hormones and then observing their response. If a sex difference is observed either developmentally or in adulthood and the sex difference is reversed by hormone treatment of the fetus or neonate, one can conclude the sex difference was hormonally "organized." If the sex difference remains, direct sex chromosome effects may contribute to the sex difference. In some instances the treatment of neonatal males with hormone blockers has no effect. This can be due to the masculinizing effects of prenatal testicular secretions, and therefore cannot be disrupted by postnatal blockade of hormone effects. This possibility can be further explored by prenatal treatments or other tools (e.g., gene knock-out mice).

2010), or so-called "experiments of nature" (Hines, 2010) in which individuals are developmentally exposed to exaggerated amounts of steroid (i.e., congenital adrenal hyperplasia) or are insensitive to or produce inadequate amounts of steroid (i.e., androgen insensitivity, silencing mutations in genes for ER or aromatase). Nonetheless, in any study a comprehensive analysis would include assessment of both developmental and adult hormonal effects, but this is often neither practical nor necessary.

\section{Hormones and the} estrous/menstrual cycle

The challenge of including hormones as a variable in an experimental design is probably the single biggest factor that de- ters the exploration of sex differences in the brain, a situation made worse by the perceived confounds of female hormonal cyclicity. Indeed, cyclical hormonal changes may either enhance or disguise a sex difference, but studying this is not prohibitively difficult. A reasonable first approach is to ignore (at least temporarily) the reproductive state and instead determine whether there is a basic sex difference in one male group versus one female group. Indeed, for some phenotypes there are no sex differences in variability of phenotype measured in this manner, despite an important hormonal modulation of the endpoint of interest, as has been proven the case in the study of pain (Mogil and Chanda, 2005). If, however, cycle stage is an important modulator of the endpoint being assessed, the intragroup variability may be greater in females than in males (although this is a not necessarily the case because there are sources of variability in males as well, such as the establishment of dominance hierarchies during group housing). Moreover, group-housed females can either repress each other's cyclicity or cycle synchronously, thereby disguising the effect of the estrous cycle. There are instances in which the cycle stage can profoundly influence the detection of a sex difference such that at one phase females are on average higher than males but at another phase are significantly lower (see McCarthy and Konkle, 2005 for specific examples). Strategies for assessing the role of reproductive cycle are summarized in Figure 3. 


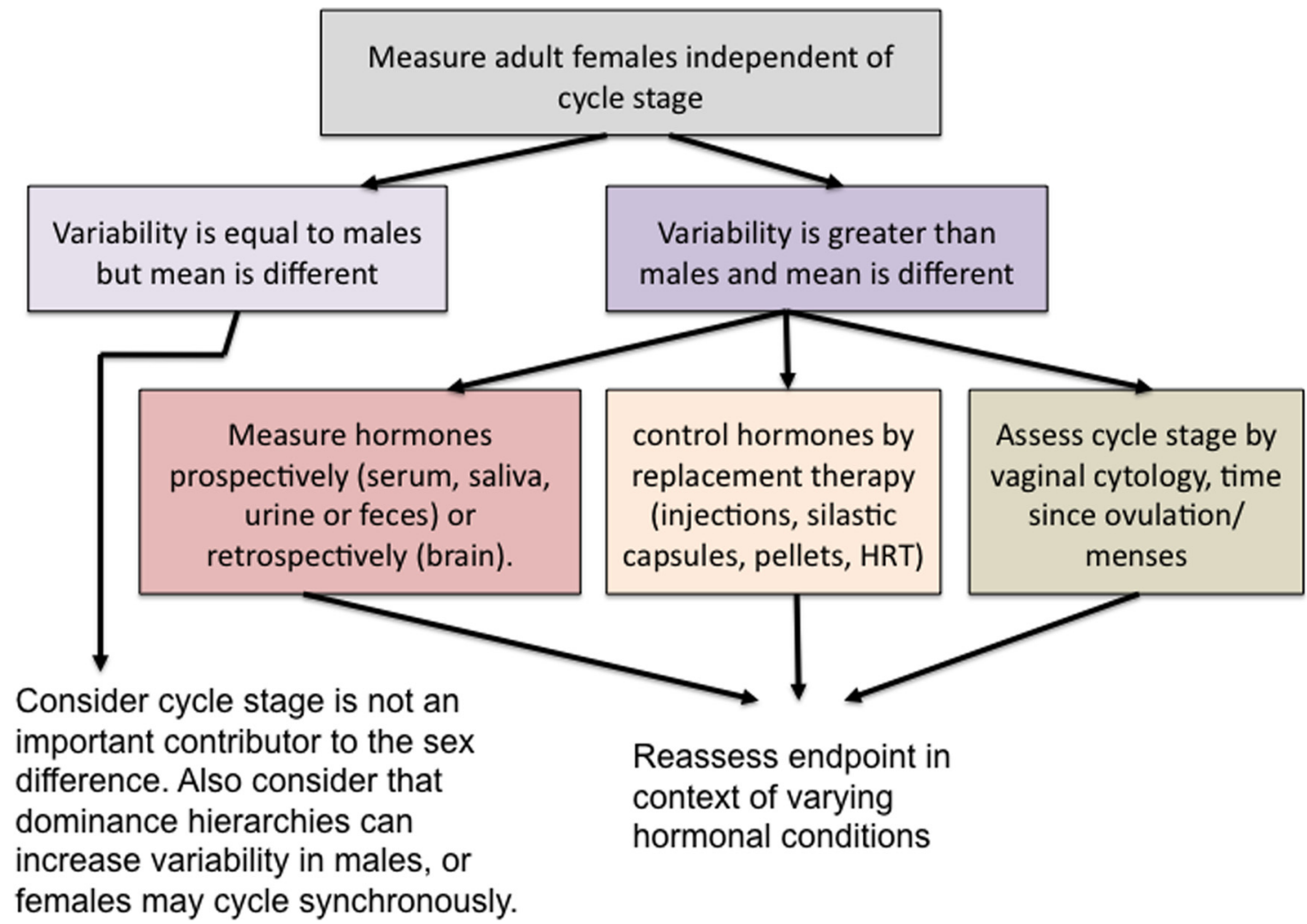

Figure 3. Impact of female cyclical reproduction on sex differences. Many endpoints with robust sex differences are not modulated by reproductive cycle stage, and a strategy is to assume this is the case until proven otherwise. A simple comparison of gonadally intact males and females will reveal whether there is greater variability in the females compared with the males. If so, this suggests that reproductive cycle stage might be important. There are various approaches that can be used to either determine the status of naturally cycling females or to provide them with a standard regimen of exogenous hormones. If the variability in females does not differ from that in males, this does not necessarily mean there is no role for the estrous cycle. Variability in males may be increased by unanticipated factors such as dominance status during group housing. Conversely, variability in females may be reduced because of either suppression or synchronization of reproductive cycles within group-housed females. Because assessing the stage of the cycle can be laborious, a simpler strategy may be to first determine which hormone(s) when given exogenously alters the endpoint, and then decide whether further assessing estrous cycle stage is worthwhile.

\section{Developmental versus adult origins}

The importance of early life programming pervades all of neuroscience but is perhaps best exemplified in the profound impact of hormones on the developing brain to "organize" or "program" the brain as male or female across the life span. Many sex differences are developmentally organized and then activated, or revealed, by the action of adult steroids, but this is not always the case. Moreover, one can never assume that there is a time point when there are no sex differences. Even primary cell cultures of neural cells from an early age show sex differences (Carruth et al., 2002; Nuñez and McCarthy, 2009). In addition, sex differences in adulthood are frequently traced to developmental origins. Whether understanding those origins is important depends on the experimental goals, but awareness of them is essential for interpretation of any results involving sex differences. Researchers may be deterred from studying development by the smaller size of the brain, the hormonal complexities of pregnancy, or lack of familiarity with manipulating newborns.
In reality, none of these is particularly problematic, and in rodent models the procedures are well established. Potential trouble spots are the dose and route of hormone administration and discerning which hormones are mediating the effects.

Unlike drugs for which doses in neonates can be scaled down from adults as a function of body weight, steroids are impacted by circulating binding globulins that are present in newborns but not adults. Moreover, some steroids are both a primary ligand of receptors and a metabolic precursor to other biologically active steroids. In rats and mice, testosterone exerts masculinizing effects on the brain and spinal cord, but testosterone is also converted to estradiol by aromatization, and this steroid exerts distinct masculinizing effects. Some endpoints are responsive only to estrogens, others only to androgens, while still others seem to require both. You can distinguish these possibilities by using non-aromatizable androgens, direct administration of estrogens, inhibitors of aromatization, or selective steroid receptor antagonists. Mutant mice that lack specific functional steroid receptors can also help distinguish the receptors that mediate the steroid effects, although a complication is that receptor knock-outs often do not allow one to discriminate between neonatal and adult effects of the hormone. Because of the potent masculinizing effects of estrogens, rodents have evolved a protective mechanism against the high circulating levels of this steroid in the pregnant dam in the form of $\alpha$-fetoprotein, a steroid-binding globulin that sequesters estrogens in the circulation of the fetus and prohibits (perhaps selectively) its entry into the brain. As a result, when studying the masculinizing effects of estradiol on the neonatal rodent brain, doses need to be as much as 10 times higher than that given to the adult. In primates, the dominant masculinizing hormones are androgens. Dosage is less of an issue in this case since $\alpha$-fetoprotein does not bind androgens and therefore does not block masculinization. Details on the administration of exogenous hormones and quantification of endogenous 
hormones and phases of the female reproductive cycle can be found in the work of Becker et al. (2005).

\section{Detecting sex chromosome effects}

Serious consideration of the potential for genetic contributions to sex differences in the brain is relatively new to the scene. The previous hegemony of hormones was the result of a combination of factors, not the least of which were technical difficulties of separating hormonal and genetic influences. A limited tool set is now available, limited in that it is mostly restricted to mice, but information gained provides a springboard for investigation of other animal models and humans.

The Four-Core-Genotypes model consists of genetically modified mice in which the testis-determining gene, Sry, which initiates testicular development from the bipotential gonad, has been moved from the Y chromosome to an autosome (Fig. 4). This produces XX mice that develop testes as well as XY mice that lack Sry and therefore develop ovaries. Comparison of these genotype/gonad phenotype-reversed animals to those in which genotype and gonads are matched distinguishes between sex differences directly driven by $\mathrm{X}$ or $\mathrm{Y}$ genes, versus those driven by hormonal products of the gonads. To date, this model system has confirmed the supremacy of hormones for most of the first type of sex differences, sex dimorphisms directly relevant to reproduction, but has revealed a genetic basis to several of the second type of sex differences, those related to social behavior, habit formation and nociception (Arnold and Chen, 2009). Similar conclusions were found in a parallel approach in which SF-1 knockout mice develop without gonads; in this model, neural sex differences directly associated with reproduction were largely, but not completely, absent in agonadal $\mathrm{XX}$ versus XY mice, but others persisted (Büdefeld et al., 2008). Mice lacking functional steroid receptors or synthetic enzymes further expand the arsenal of models for separating hormonal from genetic effects.

A second approach to study the genetics of sex differences in the brain is to study the effects of manipulating specific sex chromosome genes that are candidate factors for causing sex differences in phenotype. The $\mathrm{Y}$ chromosome is small and encodes few genes, whereas the $\mathrm{X}$ chromosome is large and gene-rich. The majority of $\mathrm{X}$ genes are not expressed at a higher level in females because of the transcriptional silencing of most of one $\mathrm{X}$

\section{Four-Core-Genotypes}

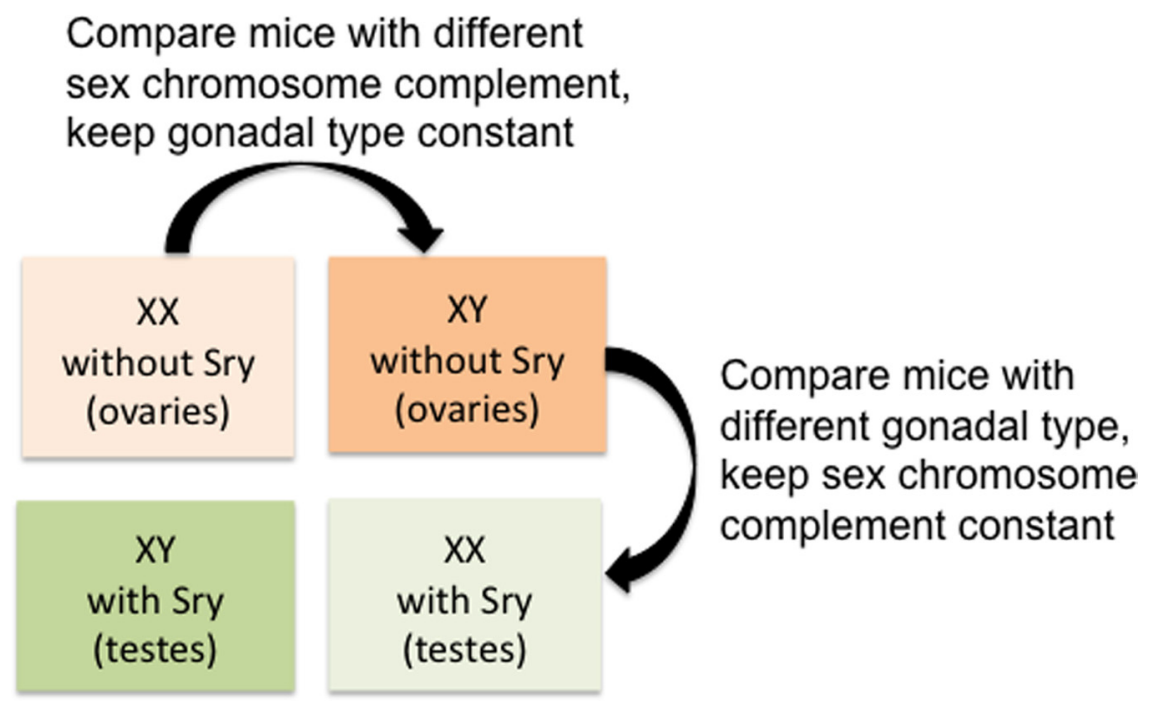

Figure 4. One strategy for detecting a "direct sex chromosome effect" on a sex difference. The importance of genetic effects to sex differences in the brain is becoming increasingly evident, and there is now a strong strategy with which to study them. The advent of the Four-Core-Genotypes mouse model provides an opportunity to compare mice of the same sex chromosome complement (XX versus $\mathrm{XX}$ ) but of the opposite gonadal phenotype (ovaries versus testes). Thus, if an endpoint varies by gonadal phenotype, it is considered hormonal in origin, and if it varies by chromosome complement, it is considered genetic in origin. There is also the potential for interactive effects between gonadal phenotype and chromosome complement.

chromosome in each XX cell. Nevertheless, some $\mathrm{X}$ genes escape inactivation and may be expressed more in females than males, or may have a parental imprint that leads to sex differences in level of expression. It is possible to test directly the role of such genes in sexual differentiation via genetic manipulation in vivo or in vitro. This approach has already proven fruitful in the discovery that Sry is expressed in dopamine neurons of the substantia nigra and has male-specific functional effects (Dewing et al., 2006). Parent-of-origin allelic expression, also called parental imprinting, in which the activity of select genes varies depending upon whether it resides on the chromosome derived from the mother versus the father can also differ in males and females, raising interesting questions about the convergence of sex-specific factors and parent-of-origin effects (Gregg et al., 2010).

\section{Conclusions}

The arguments for studying sex differences in the brain are as follows. (1) There is compelling evidence of pervasive and robust differences between males and females in both normal and pathological conditions. (2) The number of published studies limited to males remains stunningly and stubbornly high (Zucker and Beery, 2010). Scientific conclusions based on the study of one sex could have limited value in understanding some phenomena in the other sex. Thus, females deserve more study. (3) In addition, direct comparison of the two sexes is beneficial because of the unique perspective it offers. Indeed, it is seldom acknowledged that comparison of males and females has provided the critical spark to igniting widespread investigation of fundamental phenomenon. This is true for the field of adult neurogenesis, which arguably began with the study of sex differences in the song bird brain (Nottebohm and Liu, 2010); hormonal modulation of programmed neuronal cell death, which began with the discovery of the androgen-sensitive spinal nucleus of the bulbocavernosus of the spinal cord (Nordeen et al., 1985); neurosteroidogenesis, which started with the Aromatization Hypothesis (Naftolin et al., 1975); prostaglandin-mediated synaptogenesis which induces masculinization of sexual behavior (Amateau and McCarthy, 2004); and many more mechanisms likely waiting to be discovered. Moreover, in brain diseases that are sexually dimorphic, one sex is protected from disease more than the other. Thus, identifying the sex-specific protective agents could lead to better understanding of potential therapies, or identification of new drug targets. Our goal in presenting this Toolbox 
is to offer guidance and encouragement for those wishing to study sex differences.

\section{References}

Abel KM, Drake R, Goldstein JM (2010) Sex differences in schizophrenia. Int Rev Psychiatry 22:417-428.

Amateau SK, McCarthy MM (2004) Induction of PGE(2) by estradiol mediates developmental masculinization of sex behavior. Nat Neurosci 7:643-650.

Arnold AP, Wade J, Grisham W, Jacobs EC, Campagnoni AT (1996) Sexual differentiation of the brain in songbirds. Dev Neurosci 18:124136.

Arnold AP, Burgoyne PS (2004) Are XX and XY brain cells intrinsically different? Trends Endocrinol Metab 15:6-11.

Arnold AP, Chen X (2009) What does the "four core genotypes" mouse model tell us about sex differences in the brain and other tissues? Front Neuroendocrinol 30:1-9.

Baron-Cohen S, Knickmeyer RC, Belmonte MK (2005) Sex differences in the brain: implications for explaining autism. Science 310:819823.

Becker JB, Breedlove SM, Crews D, McCarthy MM (2002) Behavioral endocrinology, Ed 2. Cambridge, MA: MIT.

Becker JB, Arnold AP, Berkley KJ, Blaustein JD, Eckel LA, Hampson E, Herman JP, Marts S, Sadee W, Steiner M, Taylor J, Young E (2005) Strategies and methods for research on sex differences in brain and behavior. Endocrinology 146:1650-1673.

Beery AK, Zucker I (2011) Sex bias in neuroscience and biomedical research. Neurosci Biobehav Rev 35:565-572.

Beiko J, Lander R, Hampson E, Boon F, Cain DP (2004) Contribution of sex differences in the acute stress response to sex differences in water maze performance. Behav Brain Res 151:239-253.

Breedlove SM (2010) Minireview: Organizational hypothesis: instances of the fingerpost. Endocrinology 151:4116-4122.

Büdefeld T, Grgurevic N, Tobet SA, Majdic G (2008) Sex differences in brain developing in the presence or absence of gonads. Dev Neurobiol 68:981-995.

Cahill L (2006) Why sex matters for neuroscience. Nat Rev Neurosci 7:477-484.

Carruth LL, Reisert I, Arnold AP (2002) Sex chromosome genes directly affect brain sexual differentiation. Nat Neurosci 5:933-934.

Dao DT, Mahon PB, Cai X, Kovacsics CE, Blackwell RA, Arad M, Shi J, Zandi PP, O’Donnell
P, Knowles JA, Weissman MM, Coryell W, Scheftner WA, Lawson WB, Levinson DF, Thompson SM, Potash JB, Gould TD (2010) Mood disorder susceptibility gene CACNA1C modifies mood-related behaviors in mice and interacts with sex to influence behavior in mice and diagnosis in humans. Biol Psychiatry 68:801-810.

De Vries GJ (2004) Minireview: Sex differences in adult and developing brains: compensation, compensation, compensation. Endocrinology 145:1063-1068.

De Vries GJ, Simerly RB (2002) Anatomy, development and function of sexually dimorphic neural circuits in the mammalian brain. In: Hormones, brain and behavior, Vol 4 (Pfaff DW, Arnold AP, Etgen AM, Fahrbach SE, Rubin RT, eds), pp 137-192. New York: Academic.

De Vries GJ, Rissman EF, Simerly RB, Yang LY, Scordalakes EM, Auger CJ, Swain A, LovellBadge R, Burgoyne PS, Arnold AP (2002) A model system for study of sex chromosome effects on sexually dimorphic neural and behavioral traits. J Neurosci 22:9005-9014.

Dewing P, Chiang CW, Sinchak K, Sim H, Fernagut PO, Kelly S, Chesselet MF, Micevych PE, Albrecht KH, Harley VR, Vilain E (2006) Direct regulation of adult brain function by the male-specific factor SRY. Curr Biol 16:415-420.

Forger NG (2009) The organizational hypothesis and final common pathways: Sexual differentiation of the spinal cord and peripheral nervous system. Horm Behav 55:605-610.

Gregg C, Zhang J, Butler JE, Haig D, Dulac C (2010) Sex-specific parent-of-origin allelic expression in the mouse brain. Science 329: 682-685.

Hines M (2010) Sex-related variation in human behavior and the brain. Trends Cogn Sci 14:448-456.

Jazin E, Cahill L (2010) Sex differences in molecular neuroscience: from fruit flies to humans. Nat Rev Neurosci 11:9-17.

McCarthy M, De Vries G, Forger N (2009) Sexual differentiation of the brain: Mode, mechanisms and meaning. In: Hormones, brain and behavior, Vol 3 (Pfaff D, Arnold AP, Etgen AM, Fahrbach SE, Rubin RT, eds), pp 1707-1744. San Diego: Academic.

McCarthy MM, Arnold AP (2011) Reframing sexual differentiation of the brain. Nat Neurosci 14:677-683.

McCarthy MM, Konkle AT (2005) When is a sex difference not a sex difference? Front Neuroendocrinol 26:85-102.

Mogil JS, Chanda ML (2005) The case for the inclusion of female subjects in basic science studies of pain. Pain 117:1-5.

Morris JA, Jordan CL, Breedlove SM (2004) Sexual differentiation of the vertebrate nervous system. Nat Neurosci 7:1034-1039.

Naftolin F, Ryan KJ, Davies IJ, Reddy VV, Flores F, Petro Z, Kuhn M, White RJ, Takaoka Y, Wolin L (1975) The formation of estrogens by central neuroendocrine tissues. Recent Prog Horm Res 31:295-319.

National Institute of Mental Health (2011) Sex differences in brain, behavior, mental health and mental disorders. Workshop. Bethesda, MD, February. http://www.nimh.nih.gov/ research-funding/scientific-meetings/2011/sexdifferences-in-brain-behavior-mental-healthand-mental-disorders/index.shtml.

Nordeen EJ, Nordeen KW, Sengelaub DR, Arnold AP (1985) Androgens prevent normally occurring cell death in a sexually dimorphic spinal nucleus. Science 229:671-673

Nottebohm F, Liu WC (2010) The origins of vocal learning: new sounds, new circuits, new cells. Brain Lang 115:3-17.

Nuñez JL, McCarthy MM (2009) Resting intracellular calcium concentration, depolarizing GABA and possible role of local estradiol synthesis in the developing male and female hippocampus. Neuroscience 158:623-634.

Perrot-Sinal TS, Kostenuik MA, Ossenkopp KP, Kavaliers M (1996) Sex differences in performance in the Morris water maze and the effects of initial nonstationary hidden platform training. Behav Neurosci 110:1309-1320.

Pfaff DW, Arnold AP, Etgen AM, Fahrback SE, Rubin RT (2002) Hormones, brain and behavior. San Diego: Academic.

Shors TJ, Chua C, Falduto J (2001) Sex differences and opposite effects of stress on dendritic spine density in the male versus female hippocampus. J Neurosci 21:6292-6297.

Simerly RB (2002) Wired for reproduction: Organization and development of sexually dimorphic circuits in the mammalian forebrain. Annu Rev Neurosci 25:507-536.

Sisk CL, Zehr JL (2005) Pubertal hormones organize the adolescent brain and behavior. Front Neuroendocrinol 26:163-174.

Wade J, Arnold AP (2004) Sexual differentiation of the zebra finch song system. Ann N Y Acad Sci 1016:540-559.

Wizemann TM, Pardu ML (2001) Exploring the biological contributions to human health. Does sex matter? Washington DC: National Academies.

Zucker I, Beery AK (2010) Males still dominate animal studies. Nature 465:690. 